\title{
INTELIGENCIA ARTIFICIAL COMO HERRAMIENTA DE INNOVACIÓN EN LA MEDICINA
}

\author{
AUTORES: $\quad$ Lino Solís Yanina Joselyn ${ }^{1}$ \\ Reyes Mejía Daisy Cristina² \\ Tóala Arias Franklin Jimmy ${ }^{3}$ \\ Zambrano Chica Julissa Rafaela ${ }^{4}$ \\ DIRECCIÓN PARA CORRESPONDENCIA: (yanina7470@unesum.edu.ec)
}

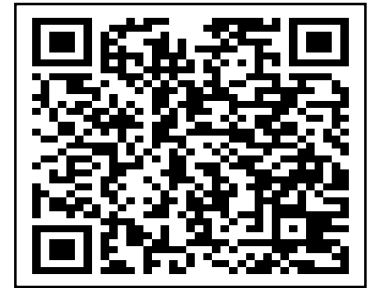

Fecha de recepción: 12/02/2021

Fecha de aceptación: 10/08/2021

\section{RESUMEN}

Esta investigación se llevó acabo para presentar la existencia y efectividad de soluciones que brinda la inteligencia artificial, los avances que esta conlleva y los recursos que nos brinda, debido a que en un futuro la inteligencia artificial será un tema de impacto en la sociedad, evolucionando desenfrenadamente su crecimiento, ofreciendo un porcentaje significativo en bienestar y salud, causando que los pacientes proporcionen una respuesta optimista en cuanto a las posibilidades de supervivencia con el que contaran. Se define como objetivo garantizar la enseñanza-aprendizaje en los estudiantes causando que tomen mayor interés en ámbitos como estos, que son de gran ayuda e interés, no solo en ámbitos medicinales sino en campos como la educación creando con ella el desarrollo de softwares e incluso también dirigido al sector inmobiliario, musical, entre otros. En base a esta investigación realizada se estableció la implementación de métodos científicos como el método de inducción - deducción y análisis síntesis, los cuales contribuyeron al desarrollo y capacidad de solucionar las diversas dudas existentes en cuanto al uso de la inteligencia artificial en la medicina. Se obtuvo como resultado final que los estudiantes obtuvieron los conocimientos necesarios y aclararon sus dudas en cuanto a la implementación positiva que llevara a cabo la inteligencia artificial, historia, sus ventajas y desventajas. Se concluye que la inteligencia artificial no solo está enfocada en la medicina sino en diversos campos, sean administrativos, comerciales, entre otros que a futuro serán de beneficio mundial, brindando mejores y mayores soluciones al ser humano.

\footnotetext{
${ }^{1}$ Estudiante de la carrera de Tecnología de la Información. Universidad Estatal del Sur de Manabí. Jipijapa - Manabí - Ecuador, Bachiller en Ciencias Unidad Educativa Fiscal "Cascol” Cascol - Manabí - Ecuador. linoyanina7470@unesum.edu.ec

${ }^{2}$ Estudiante de la carrera de Tecnología de la información. Universidad Estatal Del Sur De Manabí. Jipijapa Manabí - Ecuador, Bachiller Producción Agropecuaria en la Unidad Educativa Fiscal SUGRE Jipijapa - Manabí Ecuador. reyes-daisy6050@unesum.edu.ec

${ }^{3}$ Docente de carrera Tecnologías de la Información en la Universidad Estatal del Sur de Manabí Jipijapa-ManabíEcuador. franklin.toala@unesum.edu.ec

${ }^{4}$ Estudiante de la carrera Tecnología de la Información. Universidad Estatal del Sur de Manabí. Jipijapa - Manabí Ecuador, Bachiller en Servicios de Restaurant y Bares Unidad Educativa Fiscal “Océano Pacifíco” San Jacinto Manabí - Ecuador. zambrano-julissa3881@unesum.edu.ec
} 
Lino Solís Yanina Joselyn, Reyes Mejía Daisy Cristina, Tóala Arias Franklin Jimmy, Zambrano Ch Julissa Rafaela

PALABRAS CLAVE: Evolución; Medicina; Salud.

\title{
ARTIFICIAL INTELLIGENCE AS A TOOL FOR INNOVATION IN MEDICINE
}

\begin{abstract}
This research was carried out to present the existence and effectiveness of solutions provided by artificial intelligence, the advances that this entails and the resources it provides, because in the future artificial intelligence will be a topic of impact in society, evolving unbridled growth, offering a significant percentage of wellbeing and health, causing patients to provide an optimistic response as to the chances of survival they will have. It is defined as an objective to ensure teaching-learning in students causing them to take greater interest in areas such as these, which are of great help and interest, not only in medicinal areas but in fields such as education creating with it the development of softwares and even also directed to the real estate sector, music, among others. Based on this research, the implementation of scientific methods was established, such as the induction - deduction and analysis - synthesis method, which contributed to the development and ability to solve the various doubts regarding the use of artificial intelligence in medicine. The final result was that the students obtained the necessary knowledge and clarified their doubts regarding the positive implementation that artificial intelligence, history, its advantages and disadvantages will carry out. It is concluded that artificial intelligence is not only focused on medicine but in various fields, be they administrative, commercial, among others that in the future will be of global benefit, providing better and greater solutions to the human being.
\end{abstract}

KEYWORDS: Evolution; Medicine; Health.

\section{INTRODUCCIÓN}

La Inteligencia Artificial (IA) es la composición de algoritmos diseñados con el objetivo de crear máquinas que muestren capacidades semejantes a las del ser humano. Una tecnología que todavía nos resulta lejana y misteriosa, pero que desde hace unos años se está anexando a nuestro presente diariamente a todas horas. Radica en la capacidad de ejecutar innovaciones positivas para alcanzar los fines que se pretenden, dando soluciones mayoritarias a nivel mundial y diversos aspectos. (iberdrola, s.f.)

De acuerdo con (Moreno, 2019) "La medicina y la inteligencia artificial son dos sectores que están condenados a entenderse”. La inteligencia artificial en la salud ya está implementada y aplicada con excelentes resultados en la salud Española, para ayudar y brindar mejores soluciones a los médicos en la toma de decisiones diagnósticas y de tratamientos medicinales, previniendo efectos adversos y detección de mutaciones genéticas, pero esto no solo se da en la medicina española, existen países como China, Estados Unidos, Francia y Reino Unido que implementan la inteligencia artificial y que no se descarta una anexo de esta en temas de salud.

Dado a los grandes resultados en pruebas, gracias a los algoritmos de aprendizaje automático se logran obtener diagnósticos más claros y precisos, para así detectar síntomas de las diversas enfermedades que acarrean en el ser humano, desde una enfermedad tratable hasta las más graves antes de que estas se vuelvan intensas y sea demasiado tarde tratarlas. Por muy preparados que

84 UNESUM-Ciencias. Publicación cuatrimestral. Vol. 5, Año 2021, No. 5 (Septiembre-Diciembre) 
pueda estar un personal de salud en una labor medicinal en concreta, sabemos que los seres humanos podemos cometer errores que sean de mayor inoportunidad y que la imperturbabilidad de una maquina inteligente jamás podría permitir, además de esto sabemos que nuestro cuerpo y sentidos deben llegar a un límite en el que ya no se nos permite ser diestros y agiles como cuando empezamos , algo que un robot si puede llegar a alcanzar. Dado que por este motivo se da el beneficio del desarrollo de la inteligencia artificial, siendo esta extremadamente esencial en un gran porcentaje de ámbitos, entre los cuales no se daría pasar por alto la medicina, ahora bien podemos decir que gracias a la herramienta de innovación de la inteligencia artificial se pueden perfeccionar los diagnósticos medicinales ampliamente y de manera segura en los centros medicinales que cuenten y deseen aportar con la implementación de estos, llegando también a brindar la oportunidad de que esta tecnología innovadora se imparta incluso en los hogares de los pacientes, quienes solo necesitaran de un smartphone o cualquier artefacto que les brinde el acceso para mantener algunos de sus parámetros de salud controlados desde la comodidad de su hogar y supervisado por un familiar capacitado para manejar esta tecnología.

Todos y cada uno de los avances tecnológicos enfocados en este sentido crecen diariamente y a pasos agigantados, logrando un giro revolucionario en la medicina comparada de hace algunos años que comparada con la que día a día evoluciona es mayoritaria y beneficiaria para los usuarios, si nos detenemos a pensar rebobinado hacia años atrás esta tecnología actual nos resultaría solo de ciencia ficción y no real. Y tanto así es el impacto con el que cuenta esta herramienta de innovación que está teniendo un interés recientemente despertado con Google, que ha creado una iniciativa calificada como Google Al Impact Challenge, con el objetivo de brindar recursos educativos gratuitos y talleres de este tema en más de cinco países. De tal manera se tiene la certeza de ampliar el acceso a recursos de este campo, los cuales pueden tener el beneficio de salvar vidas. (Martín, 2019)

\section{DESARROLLO}

\section{Historia y evolución de la inteligencia artificial}

Repasamos la trayectoria que ha tenido la inteligencia artificial, comenzando con sus precursores griegos, atravesando por Alan Turing hasta IBM Watson o los actuales avances de Google o Facebook. Debido a que la inteligencia artificial se tiende a familiarizar seguidamente con la ciencia ficción, dejo de ser relegada a las novelas y películas, esta herramienta innovadora nos encierra desde las zonas más cotidianas, como lo es la conversión de voz en texto, coches autónomos, entre otros hasta los más sobresalientes como la medicina, predicción de lesiones, entre otras. Se centra en protocolos informáticos como el análisis adelantado de datos, el proceso de lenguaje natural, la visión por ordenador y el machine learning, así como explicaba actualmente Diane Bryant, vicepresidenta ejecutiva y directora general del Data Center Group de Intel. (ticbeat, 2016)

Si bien el cine y la literatura habían idealizado una manera de anexarse con la inteligencia artificial desde siempre, no fue así hasta la década de 1950 en la que se da inicio a la búsqueda de una inteligencia artificial, de máquinas capacitadas para comprender, razonar y decidir de semejante condición que la mente humana. Una de las ultimas creación con inteligencia artificial 
Lino Solís Yanina Joselyn, Reyes Mejía Daisy Cristina, Tóala Arias Franklin Jimmy, Zambrano Ch Julissa Rafaela

se dio en el año 2017 con el llamado Libratus el cual vence al póker, fue creado en la Universidad de Carnegie Mellon (EE. UU), el software se impone a rivales humanos en un torneo múltiple de Texas hold'em para dos personas. Supone un hito por la información imperfecta que caracteriza al póker. (cice, 2018)

\section{Ventajas de la inteligencia artificial}

La inteligencia artificial es complicada por naturaleza. Monopoliza una mezcla muy compleja de informática, matemáticas y otras ciencias complejas. La programación compleja ayuda a estas máquinas a replicar las capacidades cognitivas de los seres humanos.

$\checkmark$ Reducción de errores

$\checkmark$ Exploración difícil

$\checkmark$ Aplicación diaria

$\checkmark$ Asistentes digitales

$\checkmark$ Trabajos repetitivos

$\checkmark$ Aplicaciones médicas

$\checkmark$ Sin pausas ni descansos

\section{Desventajas de la tecnología artificial}

$\checkmark$ Alto Coste

$\checkmark$ Sin Replicar Humanos

$\checkmark$ No hay mejora con la experiencia

$\checkmark$ Sin creatividad original

$\checkmark$ Desempleo

Finalizando esta herramienta de innovación en los últimos tiempos y en un futuro no muy lejano motivará aún más la investigación e implementación en muchas más áreas, como la seguridad y el control de temas no muy familiarizados con lo técnico, como la economía y el derecho, pero aun así esta tecnología tendrá efectos beneficiosos en la toda la sociedad. Cabe recalcar que existen también una cierta cantidad de armas letales que son producidas con inteligencia artificial pero que se las puede controlar con el debido manejo, debido a que los creadores de esta aseguran que es benéfica y ha sido creada para ayudar a la humanidad. (agenciauniversitariadq, 2019)

\section{MATERIALES Y MÉTODOS}

En esta investigación científica predominan métodos como el método de inducción - deducción y análisis - síntesis, que contribuyeron al desarrollo y capacidad de brindar toda la información necesaria para cubrir la falta de conocimiento en personas que se interesan con herramientas de innovación como la inteligencia artificial.

El método análisis - síntesis se realizó un diagnostico general en los estudiantes sobre el tema tratado, las ventajas que posee y las necesidades que puede llegar a cubrir una tecnología como tal.

El método de inducción - deducción el cual contribuyo para llegar al tema central del problema, que es la falta de conocimientos que poseen los estudiantes, en lo referente a herramientas innovadoras como lo es la inteligencia artificial.

\section{RESULTADOS Y DISCUSIÓN}

86 UNESUM-Ciencias. Publicación cuatrimestral. Vol. 5, Año 2021, No. 5 (Septiembre-Diciembre) 
A continuación, como resultado obtenido de la investigación realizada en una encuesta dirigida a los estudiantes de la Carrera Tecnología de la Información en la Universidad Estatal del Sur de Manabí, se logró que los estudiantes tengan conocimientos del ¿Qué es? y el ¿Para qué es? beneficiosa una herramienta innovadora como lo es la inteligencia artificial sus ventajas, desventajas y la importancia con la que cuenta como parte fundamental de la Informática, sus múltiples ramas y que cada una de ellas tiene significativas aplicaciones con respecto a lo que es la medicina, que es de gran beneficio para el ser humano. Dado esto obtuvimos que los estudiantes ya cuentan con los conocimientos adecuados y suficientes para saber en qué se utiliza la inteligencia artificial y como se podría implementar de manera adecuada para la innovación de las nuevas tendencias informáticas más que todo en el ámbito medicinal. Los estudiantes tuvieron en claro que la tecnología anterior en comparación con la actual es a gran escala innovadora y que conforme avanza esta, los conocimientos también deben avanzar amplia y definidamente, debido a que la tecnología está presente y es parte importante en cada ciclo de nuestra vida.

\section{CONCLUSIONES}

La inteligencia artificial como herramienta innovadora es también un poder que involuntariamente causa destrucción, pero es un daño que minuciosamente se puede controlar y solucionar de manera constructiva, dado que con la inteligencia artificial utilizándola de manera constructiva podemos erradicar incluso la pobreza y el hambre desarrollando de manera adecuada lo que se necesita en un ámbito de esta magnitud.

Otros de los desarrollos positivos de esta innovación es que no solo es una simple inteligencia artificial como tal esta también denota de inteligencia heurística la cual cuenta con una base que surge de la experiencia y objetivo de solucionar problemas, realizar innovaciones positivas y cuenta con rasgos típicos de un humano.

La inteligencia artificial está transformando la forma de evolucionar en el mundo, se espera que informaciones como estas sean tomadas de manera positiva por la sociedad y se vea que tenemos así una manera más de interactuar con el mundo y sus innovaciones tecnológicas.

\section{REFERENCIAS BIBLIOGRÁFICAS}

agenciauniversitariadq. (20 de 03 de 2019). Obtenido de agenciauniversitariadq: https://agenciauniversitariadq.online/inteligencia-artificial-ventajas-y-desventajas/

cice. (19 de 07 de 2018). Obtenido de cice: https://www.cice.es/noticia/historia-evolucion-la-inteligencia-artificial/

iberdrola. (s.f.). Obtenido de iberdrola: https://www.iberdrola.com/te-interesa/tecnologia/que-es-inteligenciaartificial

Martín, A. (31 de 01 de 2019). hipertextual. Obtenido de hipertextual: https://hipertextual.com/2019/01/inteligenciaartificial-salud

Moreno, P. G. (20 de 02 de 2019). efesalud. Obtenido de efesalud: https://www.efesalud.com/inteligencia-artificialmedicina-futuro

ticbeat. (15 de 09 de 2016). Obtenido de ticbeat: https://www.ticbeat.com/innovacion/la-historia-de-la-inteligenciaartificial-desde-los-origenes-hasta-hoy/ 
88 UNESUM-Ciencias. Publicación cuatrimestral. Vol. 5, Año 2021, No. 5 (Septiembre-Diciembre) 